Cahiers $d u$ MONDE RUSSE

\section{Cahiers du monde russe}

Russie - Empire russe - Union soviétique et États indépendants

$51 / 4 \mid 2010$

Sciences humaines et sociales en Russie à l'Âge d'argent

\title{
Eugene M. Avrutin, Jews and the Imperial State
}

\section{Claire Le Foll}

\section{(2) OpenEdition}

Journals

Édition électronique

URL : https://journals.openedition.org/monderusse/7445

DOI : 10.4000/monderusse. 7445

ISSN : $1777-5388$

Éditeur

Éditions de l'EHESS

Édition imprimée

Date de publication : 25 novembre 2010

Pagination : 814-818

ISBN : 978-2-7132-2316-7

ISSN : $1252-6576$

Référence électronique

Claire Le Foll, «Eugene M. Avrutin, Jews and the Imperial State », Cahiers du monde russe [En ligne], 51/4 | 2010, mis en ligne le 09 décembre 2011, consulté le 03 septembre 2022. URL : http://

journals.openedition.org/monderusse/7445; DOI : https://doi.org/10.4000/monderusse.7445

Ce document a été généré automatiquement le 3 septembre 2022.

Tous droits réservés 


\title{
Eugene M. Avrutin, Jews and the Imperial State
}

\author{
Claire Le Foll
}

\section{RÉFÉRENCE}

Eugene M. AVRUTIN, Jews and the Imperial State. Identification Politics in Tsarist

Russia. Ithaca - Londres : Cornell University Press, 2010, 216 p.

Depuis maintenant plusieurs décennies, l'historiographie des juifs de Russie a connu un renouvellement considérable. Les travaux de Steven Zipperstein, John Klier, Eli Lederhendler ou plus récemment Benjamin Nathans et ChaeRan Freeze ont ébranlé les paradigmes hérités de l'historiographie juive russe façonnée par Simon Dubnov ${ }^{1}$. Au lieu d'une histoire bipolaire qui opposait une communauté homogène à des forces centripètes délétères et qui soulignait les aspects limitatifs et discriminatoires de la législation russe, ils ont mis en évidence les contradictions internes de la communauté et le rôle des rencontres entre population juive et milieu non juif. Grâce à l'ouverture des archives, ces historiens ont montré qu'une variété de facteurs a contribué, dans la seconde moitié $\mathrm{du} \mathrm{XIX}^{\mathrm{e}}$ siècle, à rendre moins étanches les frontières entre la communauté juive traditionnelle, l'État impérial et les autres groupes sociaux et ethniques de l'Empire.

2 Le premier livre d'Eugene Avrutin sur les juifs et l'État impérial russe dévoile ainsi les aspects légaux et administratifs de cette rencontre entre juifs et non-juifs. Un travail minutieux dans les archives centrales et régionales permet à l'auteur de revenir en détail sur les innombrables règlements qui régissaient l'activité économique, le droit de résidence et de déplacement ainsi que les pratiques religieuses des juifs. Dans le sillage de Nathans et de son travail sur "l'intégration sélective" des juifs les plus productifs, Avrutin explore les chemins empruntés par les juifs ordinaires pour traverser les frontières sociales et géographiques qui étaient censées maintenir les juifs dans une zone de résidence " ghetto ». À contre- courant de l'historiographie classique qui analysait la 
législation russe uniquement en termes négatifs, l'historien veut montrer ce que la loi permettait et comment les juifs s'en accommodèrent. Ces derniers eurent recours aux pratiques bien connues de corruption ou de dissimulation d'identité, mais multiplièrent également plaintes et pétitions pour négocier et infléchir un système légal flou et injuste. Des dizaines de milliers de documents témoignent de cette communication abondante et variée des juifs avec le monde extérieur. Ce livre consiste ainsi en la réfutation vigoureuse de l'idée d'une impuissance juive face à un État absolu et une législation inéluctablement oppressive.

3 Avrutin a également l'ambition d'éclairer le fonctionnement de l'État impérial russe. Il utilise l'histoire des juifs comme une étude de cas pour montrer comment, à l'instar des autres États européens, la Russie tsariste a tenté de moderniser sa manière de gouverner les populations en les identifiant plus précisément. À partir du règne de Nicolas $\mathrm{I}^{\mathrm{er}}$, l'administration cessa progressivement de régner sur des territoires et des communautés pour mieux gérer les populations à l'aide de techniques modernes d'identification des individus. Il s'agissait de mettre en place un ordre social suffisamment unifié pour qu'il puisse embrasser la diversité impériale et fonctionner administrativement sans s'appuyer sur les institutions communautaires et religieuses. Le livre d'Avrutin montre les diverses tentatives des autorités russes pour relever ce défi - documenter l'identité des individus juifs - dans un contexte de transformation accélérée de la population juive et de l'Empire russe. Car si l'identité collective des juifs était restée stable jusqu'à la première moitié du XIX siècle en étant associée à des critères simples (langue, religion, vêtement, séparatisme communautaire), la situation se complexifia à partir des Grandes Réformes d'Alexandre II, quand la mobilité sociale, économique et géographique d'une partie de la population juive eut pour conséquence acculturation, intégration sociale, voire conversion d'un petit nombre. D'après Avrutin, la conjonction de trois obstacles aurait rendu difficile l'identification des juifs : l'impossible construction d'un ordre légal et administratif capable d'englober la diversité de l'Empire, le brouillage de la limite entre identité culturelle juive et non juive, enfin la mobilité géographique des juifs. Au fil de cinq chapitres à la fois thématiques et chronologiques, l'auteur montre comment cette ingénierie sociale se construisit et comment les juifs s'adaptèrent et négocièrent avec un système de lois et d'institutions contradictoire et souvent restrictif.

4 Les deux premiers chapitres sont consacrés à la mise en place de ce système sous Nicolas I $^{\text {er }}$ et Alexandre II. Le but de ce nouvel ordre social était de créer des outils inédits pour augmenter la « lisibilité » de la population juive et réduire son isolement et son particularisme. Il s'agissait d'abord de faire un comptage plus précis de la population. L'administration s'inquiéta en effet sous Nicolas $I^{\text {er }}$ de l'imprécision des chiffres officiels fournis par les communautés juives (kahals). Selon les estimations de l'époque, jusqu'à $25 \%$ des hommes juifs n'était pas enregistrés, ce qui entraînait un problème d'évasion fiscale et militaire. Cette inexactitude ne résultait pas seulement de la mauvaise volonté des kahals à communiquer le nombre exact de juifs relevant d'un taux d'imposition élevée, mais aussi d'autres facteurs: mobilité de la population, changements de noms, incendies et obligation d'utiliser le russe. La nécessité de moderniser les outils de gestion de la population et d'enregistrer le statut légal de chaque individu entraîna l'introduction de documents plus détaillés. La tenue d'un registre d'état civil, imposée au clergé par Pierre le Grand en 1722, devint une obligation pour les rabbins en 1835. Mais l'enregistrement des individus posa des problèmes pratiques autant que culturels et religieux car l'inscription de l'année de naissance ne faisait pas partie des coutumes religieuses et culturelles juives. Enfin, cette réforme de 
l'ordre social impérial imposa aux juifs une régulation de leurs pratiques vestimentaires. Afin de minimiser les différences culturelles et d'unifier l'Empire, le gouvernement ne se contenta pas d'abolir le kahal et de créer des écoles juives d'état, mais il força également les juifs à renoncer à leur vêtement traditionnel pour adopter un mode vestimentaire moins distinctif. Il s'agissait de supprimer le marqueur le plus visible de leur identité en le remplaçant par des documents techniques comme le passeport ou l'état civil. Cependant, la pénurie de fonctionnaires ainsi que la survivance du kahal condamnèrent à l'échec la plupart de ces réformes de la vie juive.

5 Sous Alexandre II, les enjeux et les difficultés d'enregistrement de la population ne firent que croître (ch. II). La réforme militaire de 1874 nécessitait d'avoir des registres exacts tenus désormais par un office local. Mais l'état civil continua de fait à être tenu par les autorités religieuses, dans le meilleur des cas. Le nombre de communautés qui n'avaient pas de rabbin reconnu par l'État ne cessait en effet d'augmenter du fait de l'hostilité de la population à ces rabbins officiels et de l'apparition de nouvelles communautés dans la Russie intérieure par suite de l'assouplissement des règles de résidence. Provenant de la société russe, les accusations d'évasion militaire, de corruption, de négligence et d'inefficacité chez les rabbins se multiplièrent. Mais le nombre de plaintes et de pétitions des juifs eux-mêmes dénonçant l'incurie des rabbins augmenta au fur et à mesure qu'ils prenaient conscience que leurs choix de vie (professionnel, social, éducation, résidence) dépendaient en grande partie des documents qu'ils détenaient (passeports, certificats).

Les restrictions imposées aux juifs de Russie quant à leur droit de résidence et de déplacement restent les symboles les plus frappants de l'oppression tsariste. Cependant, Avrutin nuance cette vision dans le ch. III en mettant en lumière les brèches de cette muraille légale construite autour des juifs. L'auteur commence par rappeler que les migrations vers les grandes villes de la zone et les quatre coins de la Russie intérieure s'intensifièrent avec les Grandes Réformes, grâce à la révolution des transports, au développement du capitalisme et à un assouplissement des règles. Les pogroms de 1881 conduisirent cependant à un durcissement considérable du régime des passeports et des règles de résidence pour les juifs. Les lois de mai 1882 ne les autorisaient à vivre que dans des localités urbaines. Cela aboutit à de nombreuses expulsions hors d'un certain nombre de villages et de grandes villes. Sans minimiser l'ampleur et les conséquences désastreuses des expulsions, Avrutin montre que la population juive résista par tous les moyens à leur application: pétitions, plaintes, appels envoyés aux gouverneurs de province, ou simplement refus de quitter les localités où ils n'avaient pas officiellement le droit de vivre.

Le contrôle de l'identité et du déplacement des juifs était rendu difficile par les divers procédés dont ceux-ci usaient pour dissimuler leur judéité (ch. IV). La conversion, très minime en chiffres bruts, garantissait théoriquement l'égalité légale avec les chrétiens. Cependant, l'augmentation du nombre des conversions incita le saint-synode à repérer et à dénoncer celles qui n'étaient pas authentiques. En 1903, seuls les juifs qui jouissaient déjà du droit de résidence pouvaient se convertir et vivre en dehors de la zone de résidence. À partir de 1905, l'origine juive du converti fut précisée dans le passeport afin de décourager les conversions abusives. Ainsi les juifs convertis ne pouvaient échapper à leurs origines et les demandes de changement de nom ou de suppression de la mention « juif » dans leur passeport étaient rarement satisfaites. Il existait cependant des moyens plus accessibles et moins traumatisants pour masquer leur identité sociale: l'achat de faux passeports (pour 3 ou 4 roubles), la fabrication de faux certificats officiels ou la 
corruption de fonctionnaires étaient des pratiques répandues. Les juifs qui circulaient à l'intérieur de la zone de résidence sans passeport intérieur étaient également très nombreux. Mais la proportion de juifs qui quittèrent la Russie sans autorisation de sortie ni passeport est encore plus impressionnante (entre 75 et $99 \%$ selon les estimations) et révèle combien la police et les autorités russes étaient impuissantes à contrôler leurs déplacements. Le contrôle des révolutionnaires était également très difficile sans identification photographique.

Dans un dernier chapitre passionnant, Avrutin analyse l'importance et les contradictions que le nom (juif) a revêtues à la fin de la période tsariste. Contraints d'avoir un nom de famille depuis 1804, beaucoup de juifs le modifièrent au cours du siècle pour signifier leur aspiration à l'acculturation. Malgré la politique impériale de fusion des juifs dans la société russe, le changement de nom fut interdit après 1887. Cela n'empêcha pas une hausse spectaculaire du nombre de demandes sur fond de xénophobie croissante, en particulier pendant la Grande Guerre. Cela n'empêcha pas non plus les juifs les plus en vue - marchands, financiers, businessmen - d'utiliser un nom russe dans leur vie quotidienne, malgré les amendes et peines de prison qu'ils encouraient. Le saint-synode fut aux avant-postes de cette bataille autour du nom juif. Outre son refus de changer le nom des juifs convertis, cette institution voulait que les juifs aient obligation de porter des noms juifs. À cette fin, des compilations de noms juifs et chrétiens furent publiées. D'après Avrutin, cela prouve que le nom était devenu le nouvel outil d'identification des juifs. Apparemment plus fiable que la religion, la langue ou la culture, le nom devint ainsi la technique privilégiée par l'État russe pour identifier ethniquement les juifs et tracer une frontière avec les Russes. Cependant, les critiques violentes qui s'élevèrent contre les compilations de noms juifs publiées entre 1874 et 1908 montrent qu'il n'était pas si facile de distinguer un juif seulement par son nom, d'une part car la loi juive n'interdisait pas d'utiliser des noms chrétiens, d'autre part parce que beaucoup de noms russes venaient de l'Ancien Testament.

Cet ouvrage permet donc enfin de donner sens et vie à ces milliers de documents d'archives - plaintes, requêtes, listes, dénonciations - émis par la population juive. En émaillant son récit de nombreux exemples, Avrutin nous fait ainsi rencontrer des personnages hauts en couleur appartenant à cette masse grise de juifs qui naviguaient entre zone de résidence et Russie, entre identité traditionnelle et modernité. Il nous fournit également de nombreuses clés pour saisir l'importance de ce dialogue permanent entre juifs et État impérial.

\section{NOTES}

1. Steven Zipperstein, The Jews of Odessa: A Cultural History, 1794-1881, Stanford: Stanford University Press, 1985 ; John D. Klier, Imperial Russia's Jewish Question, 1855-1881, Cambridge : Cambridge University Press, 1995 ; Eli Lederhendler, The Road to Modern Jewish Politics: Political Tradition and Political Reconstruction in the Jewish Community of Tsarist Russia, New York - Oxford: Oxford University Press, 1989 ; Benjamin Nathans, Beyond the Pale: The Jewish Encounter with 
Late Imperial Russia, Berkeley: University of California Press, 2002 ; ChaeRan Freeze, Jewish Marriage and Divorce in Imperial Russia, Hanover : University Press of New England, 2001. 